\title{
Active actinium
}

\author{
Naturally scarce but synthetically accessible, Gauthier J.-P. Deblonde and Rebecca J. Abergel discuss \\ element 89 and its emergence as a candidate radio-theranostic metal for cancer treatment.
}

n 1899, during the aftermath of Marie and Pierre Curie's resounding discovery of both polonium and radium, André-Louis Debierne - one of their French research fellows - partially isolated another new element. Ambiguities in Debierne's purification procedure from pitchblende residues almost cost him the credit for the discovery of element 89 , with Friedrich Oskar Giesel independently managing to isolate the same isotope just three years later. After some discussion, Debierne's actinium (for the Greek aktinos, meaning ray) was preferred to Giesel's emanium; although both names highlight its active alpha-particle emanations.

The discovery of Ac was a continuation of the work of the Curies, but never had the same impact as its freshly discovered radioactive neighbour, Ra. Indeed contrary to radiumbased therapies, actinium had no commercial applications at the time, and its extreme rarity in nature made it almost impossible to extract from ores - a process that is still difficult even with today's technologies.

Unlike ${ }^{226} \mathrm{Ra}$, which has a relatively long half-life $\left(t_{1 / 2}\right)$ of 1,600 years, the only naturally occurring isotopes of actinium are ${ }^{227} \mathrm{Ac}\left(t_{1 / 2}=21.8\right.$ years $)$, which comes from the decay of the already rare ${ }^{235} \mathrm{U}$, and ${ }^{228} \mathrm{Ac}$ $\left(t_{1 / 2}=6.1\right.$ hours $)$, a furtive decay product of ${ }^{232} \mathrm{Th}$. The elusive nature of Ac, combined with two world wars in the first half of the twentieth century, resulted in the knowledge of no new isotopes until 1947, though 32 have now been identified and 60 more are expected to exist. Unfortunately, this profusion of new man-made Ac isotopes synthesized since the 1950's has not produced any long-lived actinium atoms, they all actively decay in a few hours at best, with the exception of ${ }^{225} \mathrm{Ac}$ $\left(t_{1 / 2}=10\right.$ days $)$. Bulk chemical studies on actinium are therefore still dependent on the original ${ }^{227} \mathrm{Ac}$ (ref. 1). Nonetheless, progress in particle accelerators and nuclear reactions made both ${ }^{227} \mathrm{Ac}$ and ${ }^{225} \mathrm{Ac}$ more accessible

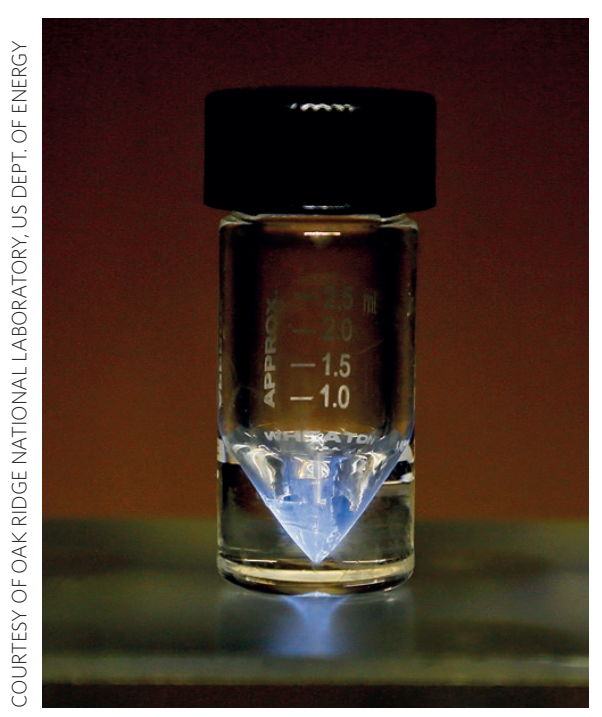

Purified ${ }^{225} \mathrm{Ac}$ emitting Cerenkov luminescence.

through neutron or proton irradiation of ${ }^{226} \mathrm{Ra}$ or ${ }^{232} \mathrm{Th}$ targets.

Beyond credit for discovery, the debate on the position of actinium in the periodic table is still highly active ${ }^{2}$. Although the name actinium would unambiguously make it the first member of the actinide family, Ac is still placed below Sc, Y and La in most periodic tables. Recent arguments tend to support its reassignment as an actual $f$-block member with irregular electronic configuration, whereas the current heaviest actinide, Lr, would be drafted to the $d$-block below Sc, Y and Lu. Regardless of whether Ac is an $f$ - or $d$-element, its chemistry has experienced a revival in the past few years ${ }^{3}$. Indeed, actinium may supersede radium due to its ongoing development in alpha-emitting radionuclide therapies. If chelated and targeted adequately, the tremendous energy released by the fourfold alpha disintegrations of ${ }^{225} \mathrm{Ac}$ could be used to surgically strike prostate, breast and bone marrow cancer tumours. In contrast to very short-lived isotopes such as ${ }^{213} \mathrm{Bi}$ $\left(t_{1 / 2}=46\right.$ minutes $)$ and ${ }^{211}$ At $\left(t_{1 / 2}=7.2\right.$ hours $)$ that have been evaluated for these applications, the day-scale decay of the now easily accessible ${ }^{225} \mathrm{Ac}$ makes it a seemingly ideal candidate for destroying tumour cells. Its final decay to the stable and non-toxic ${ }^{209} \mathrm{Bi}$ is also beneficial when compared with competitors under clinical evaluation, namely ${ }^{227} \mathrm{Th}$, ${ }^{228} \mathrm{Th}$ and ${ }^{230} \mathrm{U}$, all of which alpha-decay to stable, but toxic, $\mathrm{Pb}$ isotopes.

A rising challenge for chemists resides in the handling of ${ }^{225} \mathrm{Ac}$ intermediates and potentially harmful recoiling daughters ${ }^{221} \mathrm{Fr},{ }^{217} \mathrm{At}$, and ${ }^{213} \mathrm{Po}$. The design of in vivo stable carriers able to scavenge both actinium and its alpha cascade raises hope for highly efficient cancer therapies with no side effects. Various strategies are under development, among which the encapsulation within nanoparticles seems most promising, whereby tumour delivery of ${ }^{225}$ Ac through a lanthanide-based 'nanosafe' could neutralize metastases without leaving radioactive traces in the patient ${ }^{4}$. Finally, Cerenkov luminescence imaging of targeted tissues using the in situ decay of ${ }^{225} \mathrm{Ac}$ and its daughter products has recently brought to light the formidable theranostic potential of ${ }^{225}$ Ac-based radiopharmaceuticals ${ }^{5}$.

The quest for an efficient and silent, but traceable cancer treatment could turn into reality thanks to an element that has been largely overlooked since its discovery. Studies of actinium have taken researchers through the realms of radiochemistry, nuclear science, particle physics, medicine, and more recently nanomaterial design, underlining how Ac chemistry is as highly active as ever.

\section{GAUTHIER J.-P. DEBLONDE and} REBECCA J. ABERGEL are in the Chemical Sciences Division, Lawrence Berkeley National Laboratory, MS 70A-1150, One Cyclotron Road, Berkeley, California 94720, USA. e-mail: gdeblonde@lbl.gov; rjabergel@lbl.gov

\section{References}

1. Ferrier, M. G. et al. Nat. Commun. 7, 12312 (2016).

2. Castelvecchi, D. Nature http://doi.org/bq8s (2015).

3. Miederer, M., Scheinberg, D. A. \& McDevitt, M. R. Adv. Drug Deliv. Rev. 60, 1371-1382 (2008).

4. McLaughlin, M. F. et al. PLOS One 8, e54531 (2013)

5. Pandya, D. N. et al. Theranostics 6, 698-709 (2016). 